\title{
Internet-Delivered Psychological Treatment Options for Panic Disorder: A Review on Their Efficacy and Acceptability
}

\author{
Jennifer Apolinário-Hagen $\bowtie$ \\ Department of Health Psychology, Institute of Psychology, University of Hagen, Hagen, Germany
}

Background Internet-delivered psychological treatments have been suggested as a chance to expand the access to professional help. However, little is known about the usefulness of different support formats and approaches of digital treatments for panic disorder among clinicians.

Objective This narrative review aimed to explore the recent evidence base on the efficacy and acceptability of different internet-delivered treatments for adults with panic disorder.

Methods A systematic search in electronic databases (Pubmed/Medline, PSYNDEX) and a hand search were performed to identify articles on randomized controlled trials published within the past five years (2012/12/10-2017/12/12) in English peer-reviewed journals.

Results Eight studies (1,013 participants) involving 10 interventions met the inclusion criteria. Nine interventions were primarly based on Cognitive Behavioral Therapy principles. Most interventions were effective, when compared to a control condition (6 of 8 comparisons). Minimal guidance was associated with improved outcomes in one study and adherence in two studies (3 comparisons). Furthermore, no differences were found based on treatment approach ( 2 comparisons). Regarding acceptability, the attrition rates were moderate to high, ranging from $9.8 \%$ to $42.1 \%$ of randomized participants. Adherence rates also varied largely $(7.8-75 \%)$, whereas participant satisfaction of program completers was assessed overall high (5 studies).

Conclusion Diverse effective internet-delivered treatments are available for the self-management of panic symptoms. Especially selfguided and transdiagnostic Cognitive Behavioral Therapy approaches appear being efficient options for the dissemination in routine care. However, due to the limited evidence base, further efforts are required to improve the actual uptake of internet-delivered treatments and identify moderators of outcomes.

Psychiatry Investig 2019;16(1):37-49

Key Words Panic disorder, Anxiety disorder, eHealth, Cognitive behavior therapy, Internet.

\section{INTRODUCTION}

Anxiety disorders considerably contribute to the global burden of mental disease due to their high incidence, tendency to chronification and comorbidity. ${ }^{1-3}$ Panic disorder (PD), respectively $\mathrm{PD}$ with agoraphobia (PDA), is the most common anxiety disorder during early adulthood, ${ }^{3}$ with an estimated lifetime prevalence of $1.7 \%$, and $80.4 \%$ of persons with PD having a lifetime comorbid mental disorder. ${ }^{4}$ Since recurrent panic attacks and worries decrease subjective well-being, qual-

Received: March 30, 2018 Revised: May 29, 2018

Accepted: June 26, 2018

$\triangle$ Correspondence: Jennifer Apolinário-Hagen, $\mathrm{PhD}$

Department of Health Psychology, Institute of Psychology, Fernuniversität in Hagen (University of Hagen), Universitätsstraße 33, 58097 Hagen, Germany

Tel: +49-2331-987-2272, Fax: +49-2331-987-1047

E-mail: jennifer.apolinario-hagen@fernuni-hagen.de

(a) This is an Open Access article distributed under the terms of the Creative Commons Attribution Non-Commercial License (https://creativecommons.org/licenses/bync/4.0) which permits unrestricted non-commercial use, distribution, and reproduction in any medium, provided the original work is properly cited. ity of life and psychosocial functioning, ${ }^{5}$ untreated PD is associated with a higher risk of disability. ${ }^{6}$

In addition to pharmacotherapy, different evidence-based psychological treatment options are available for $\mathrm{PD},{ }^{6,7}$ such as , Cognitive Behavioral Therapy (CBT). ${ }^{8}$ Yet, persistent treatment gaps ${ }^{9}$ hamper the access to such treatments in health care. Moreover, especially young adults with anxiety appear to prefer online self-help over face-to-face services. ${ }^{10}$ Internetdelivered psychological treatments have been proposed as an option to expand the access to therapy ${ }^{11}$ as they provide additional options for the dissemination of psychological treatments for persons not able or not willing to use face-to-face services. ${ }^{12,13}$ Several internet treatments are available, differing in principle, support format and treatment approach. While most internet treatments are based on the principles of $\mathrm{CBT}^{14}$ Mindfulness Cognitive Therapy, ${ }^{15,16}$ Acceptance Commitment Therapy (ACT) $)^{17}$ and psychodynamic therapy ${ }^{18}$ have also been transferred to Internet-delivery. 
In recent years, most research efforts and advances were associated with internet-delivered CBT (iCBT) programs. ICBT for PD delivers the same components like traditional face-to-face CBT, such as psychoeducation, cognitive restructuring, de-arousal and exposure, as online self-help format. ${ }^{19}$ Like CBT, iCBT can reduce the frequency of panic attacks and avoidance behavior in most patients, whereas psychoeducation alone and pure self-help are suitable options for milder, recently manifested PD forms. ${ }^{20}$ As common support formats, self-guided and clinician-guided internet treatments are provided, whereas the latter typically combine structured self-help content with minimal scheduled support, for instance, via email or telephone. ${ }^{21,22}$ Although most treatment approaches are disorder-specific, transdiagnostic tailored internet treatments are of increasing relevance as they allow for simultaneous treatment ${ }^{23}$ and thereby address comorbidity and overlapping symptoms in anxiety disorders. ${ }^{24}$

Numerous randomized controlled trials (RCTs) have demonstrated the effectiveness of internet treatments for anxiety disorders. ${ }^{14,25-27}$ Furthermore, research evidence indicated an equivalent effectiveness of guided iCBT with face-to-face $\mathrm{CBT}^{28-30}$ and that iCBT for PD can be successfully provided under routine care conditions. ${ }^{31,32}$ Conversely, still little is known about specific features such as support format and treatment approach making iCBT effective. ${ }^{21}$ For instance, while some reviews indentified that guidance appears to improve therapeutic effects of iCBT for anxiety disorders. ${ }^{26,33,34}$ Others ${ }^{14}$ indicated no substantial impact. Furthermore, findings regarding the role of treatment approaches are undecided, but suggest higher effects of more established disorder-specific treatments. Nonetheless, recent meta-analyses ${ }^{35-37}$ also identified medium to large effect sizes for transdiagnostic and tailored iCBT for anxiety disorders, when compared to a wait-list control (WLC), active control conditions or care as usual (CAU). Hence, it would be interesting to determine which treatments work best for persons with PD.

Moreover, or the successful dissemination of internet treatments for PD the acceptance of patients is an important precondition..$^{15}$ Acceptability of iCBT is generally rated as high. ${ }^{38}$ For instance, a systematic review ${ }^{14}$ showed high participant satisfaction in therapist-guided iCBT for anxiety disorders. These positive findings that were collected from program completers, however, could be biased, since attrition rates are often high in studies on internet treatments for anxiety disorders. ${ }^{26}$ Adherence rates also vary largely. According to a systematic review on transdiagnostic and disorder-specific iCBT, ${ }^{35}$ adherence rates varied between $43 \%$ and $90 \%$, indicating a discrepancy between treatment satisfaction and adherence. Research has shown that guided iCBT for anxiety disorders yielded higher acceptability in terms of adherence and participant satisfaction. ${ }^{34}$ However, the relevance of such features in outcomes of internet treatments appears indecisive. Insofar, clinicians should keep themselves up-to-date of recent developments in internet-delivered therapies for PD to decide on their suitability for patients.

Considering the broad scope of systematic reviews on several anxiety disorders, ${ }^{12-14,25,26,28,30,35,36}$ the rapidly growing evidence base and the heterogeneous assessment in studies, a narrative synthesis of the literature was performed to provide a rapid overview of current internet-delivered treatment for PD. This review aims at exploring the present evidence base on 1) the effectiveness of internet treatments for PD in comparison to a control condition as well as potential differences in outcomes between support formats or treatment approaches. Another purpose was to explore 2) indicators of acceptance of internet treatments, namely attrition and adherence rates as well as participant satisfaction.

\section{METHODS}

\section{Eligibility criteria}

This narrative review included papers targeting the effectiveness of internet treatments for PD, published in English peer-reviewed journals in the past five years. Based on the PICOS tool (Participants, Intervention, Comparator, Outcome, Study design) ${ }^{39}$ the following inclusion criteria were defined:

- Participants: adults aged over 18 years, primary or secondary PD-diagnosis according to Diagnostic and Statistical Manual of Mental Disorders (DSM-IV or -V)

- Intervention: stand-alone, structured internet-delivered psychological treatment

- Comparators: (a) control condition (passive, active, CAU) or another active treatment arm with respect to (b) support format (unguided vs. guided) or (c) treatment approach (disorder-specific vs. transdiagnostic).

- Outcomes: (I) primary outcomes: reduction of panic symptom severity on a disorder-specific self-report measure at post-treatment, assessed via intention-to-treat (IIT)-analyses; (II) secondary outcomes: acceptability in terms of (a) attrition (randomized cases) and adherence rates (completion of all lessons/modules), as well as (b) participant satisfaction.

- Study design: only RCTs were included. Qualitative studies, re-examinations or secondary analyses of RCTs, study protocols and review articles were excluded.

\section{Information sources, search and study selection}

Electronic databases (PubMed/Medline, PSYNDEX) were systematically searched using the keywords (Medical Subject Headings; MeSH terms) "panic disorder" AND “internet" AND 
"therapy" to identify RCTs of the past five years (2012/12/122017/12/10). To minimize the amount of irrelevant records in the PubMed search, "randomized controlled trial" and "last five years" were applied as filters. PSYNDEX was searched over the PubPsych platform using the filters "PSYNDEX," " 5 years," "English," and "journal article." The last date searched was the 10th December 2017 for databases (PubMed, PSYNDEX) and the 29th December 2017 for additional sources [e.g., PubMedCentral (PMC), Cochrane Library, "Internet Interventions," "Journal of Medical Internet Research," reference lists of systematic reviews and meta-analyses] using further keywords such as "iCBT." Risk of bias or study quality was not assessed. As a rule of thumb, high risk of bias was assumed in studies with an attrition rate of over $20 \%$, whereas $5-20 \%$ represent a moderate risk of bias. ${ }^{40}$

\section{RESULTS}

\section{Study selection}

The systematic database search resulted in 32 records, of which 22 stem from PubMed and 10 from PSYNDEX (Figure 1). After title screen, six of 22 records identified via PubMed were excluded (i.e., study protocols, ${ }^{41-44}$ a Non-English paper, ${ }^{45}$ and a study with children ${ }^{46}$ ). Of the 10 records found through PSYNDEX, seven were duplicates and three not eligible (i.e., an off-topic conference, ${ }^{47}$ a qualitative study, ${ }^{48}$ and a review $\left.{ }^{49}\right)$. Next, the abstracts of 16 records were screened. Eight records targeting no internet-delivered treatment, ${ }^{50}$ blended treatment, ${ }^{32} \mathrm{PD}$ not as the main target ${ }^{51-53}$ and secondary analyses $^{54-56}$ were excluded. Then, after abstract reads of several papers found through hand search, four full texts were obtained that were found among related citations in PubMed, ${ }^{57}$ PMC ${ }^{19,58}$ reference lists and Cochrane Library. ${ }^{59}$ After reading 12 full texts, four publications ${ }^{58-61}$ were excluded, because outcomes reported at post-treatment were not PD-specific. Finally, eight RCTs were included.

\section{Study characteristics}

The eight included papers were published between 2013 and 2017 (Table 1). Sample sizes ranged from $n=63$ to $n=179$. A total of 1,013 participants was recruited mostly from the general population in Australia, ${ }^{19,21}$ Germany, ${ }^{23,24,62}$ Switzerland ${ }^{24}$ Austria, ${ }^{23}$ the Netherlands, ${ }^{63}$ Sweden,${ }^{64}$ and Spain, ${ }^{57}$ mainly online $e^{19,21,23,24,57,62-64}$ and traditional media advertise-

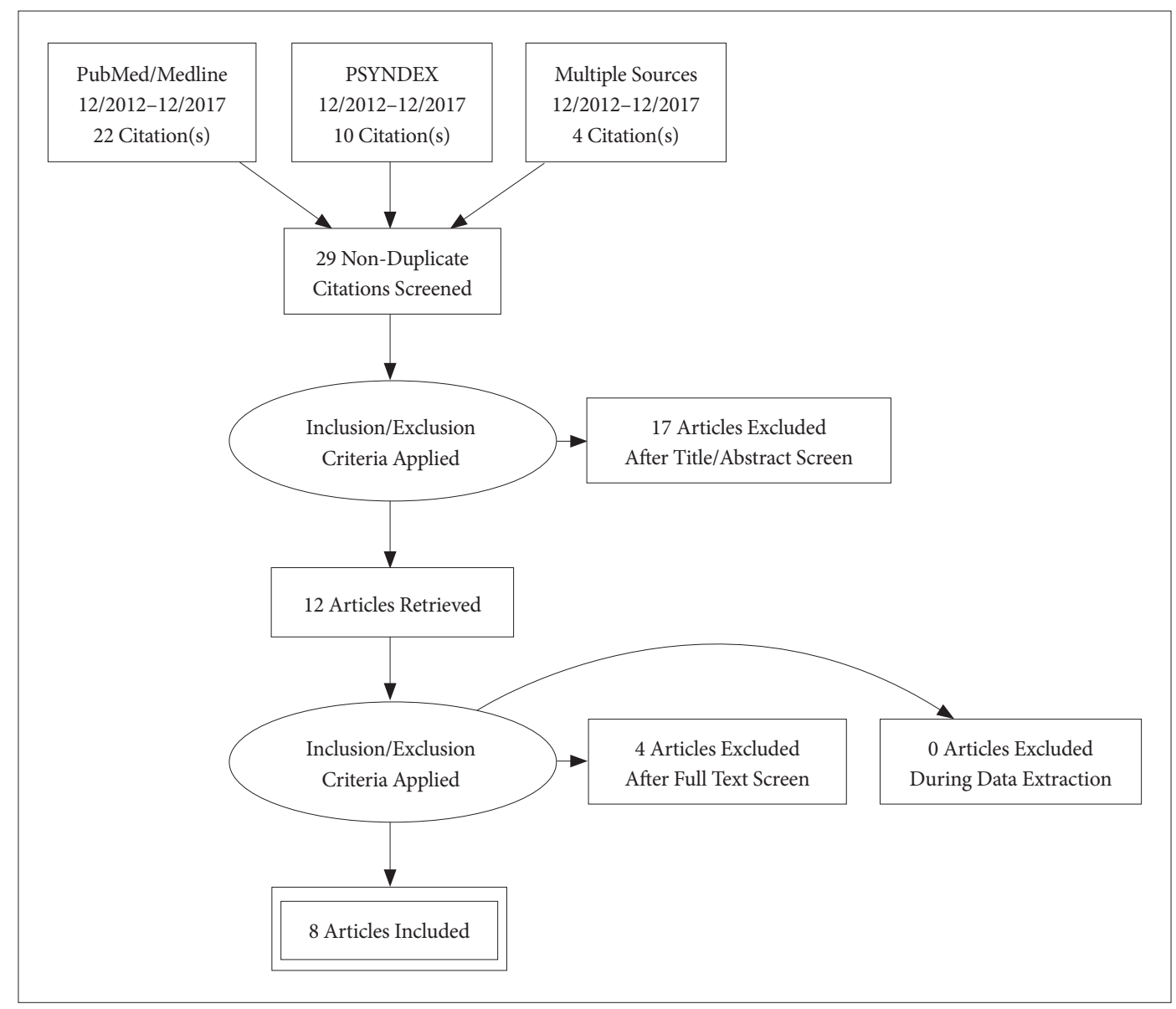

Figure 1. Flow chart diagram of study selection. 


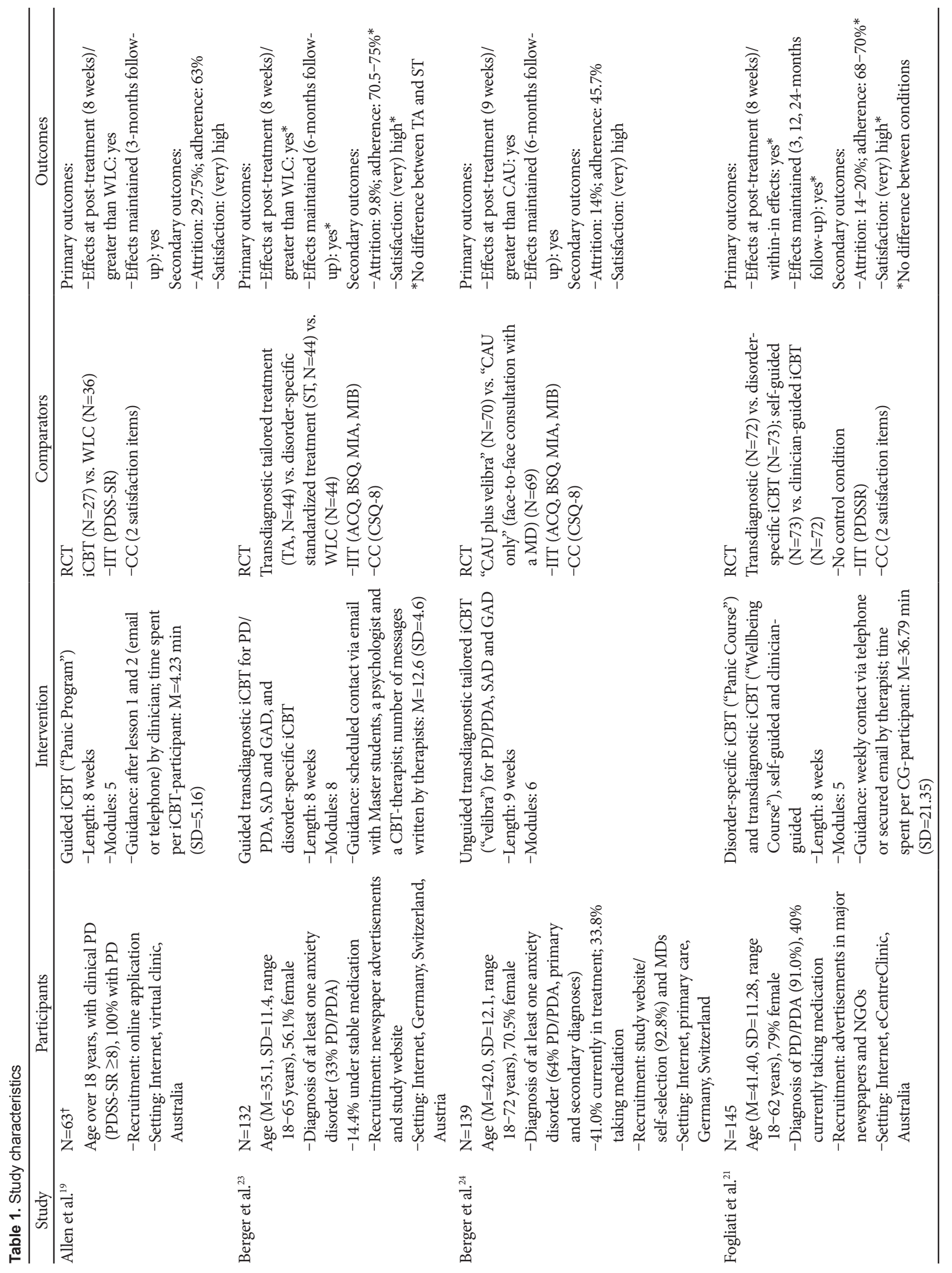




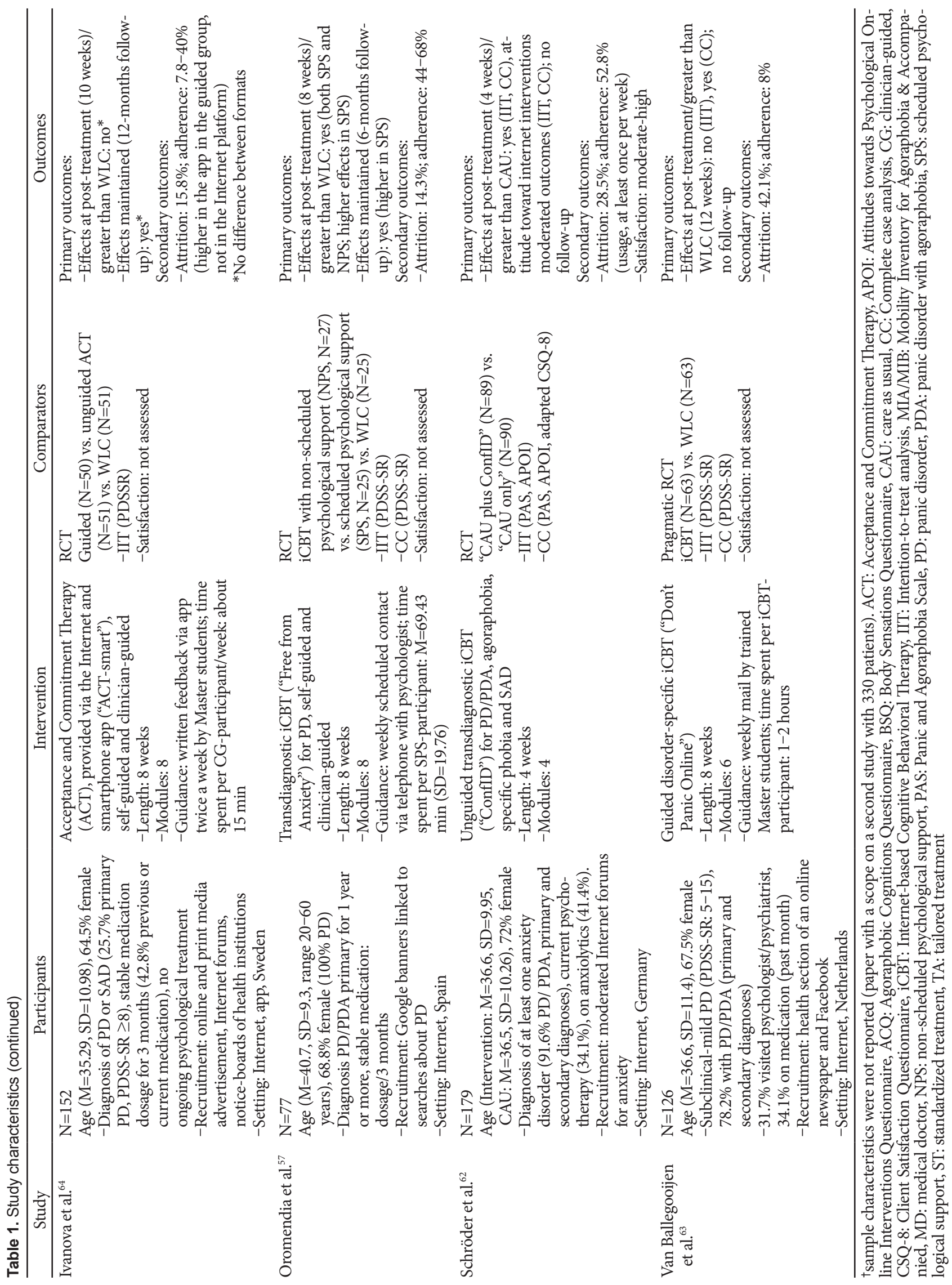


ments $^{21,23,64}$ via self-selection and in primary care. ${ }^{24}$

\section{Participants}

Participants were aged between 18-72 years (mean age: 35-42). Most were female (range $56.1^{23}-79.0 \%{ }^{21}$ ). Many had a University degree (range $31.3^{62}-57.0 \%{ }^{21}$ ) and experience with psychological treatments (reported in four studies, $53^{23}$ $77.7 \%^{24}$ ). The proportion of PD/PDA cases varied between $25.7 \%{ }^{64}$ and $100 \% .{ }^{57}$ Comorbidity was high in some studies (e.g. $\left.{ }^{62,63}\right)$.

\section{Interventions}

Out of web-based 10 interventions, four were disorder-specific $^{19,21,23,63}$ and six transdiagnostic treatments [for a summary of contents, Supplementary Table 1 (in the online-only Data Supplement)]. 21,23,24,57,64,62 Nine interventions were based on $\mathrm{CBT}^{21,23,24,57,62,63}$ and one was ACT-based with CBT elements. ${ }^{64}$ Most interventions had a length/availability of 8 weeks (range $4^{62}-12^{63}$ ), with 4 to 8 lessons. Unguided self-help $p^{21,24,57,62,64}$ was provided with automated messages and support on demand. Guidance, ${ }^{19,21,23,57,63,64}$ via email or telephone varied in the average time spent from a few minutes ${ }^{19}$ up to one or two hours (per participant/program). ${ }^{21,57,63,64}$ Therapist qualifications differed (e.g., Master students, psychologists, CBT-therapists).

\section{Comparators}

Seven RCTs compared eight active treatments against a control condition, three studies guided vs. unguided formats, and two studies transdiagnostic vs. disorder-specific approaches (i.e., 13 comparisons) at post-treatment.

\section{Outcomes}

(I) The Panic Disorder Severity Scale Self-Report version (PDSS-SR) was used in five studies, ${ }^{19,21,57,63,64}$ multiple measures [Agoraphobic Cognitions Questionnaire (ACQ), Body Sensations Questionnaire (BSQ), Mobility Inventory for Agoraphobia \& Accompanied (MIB, MIA)] in two studies ${ }^{23,24}$ and the Panic Agoraphobia Scale (PAS) in one study. ${ }^{62}$ (II) As shown in Table 1, all studies reported attrition (9.8-42.1\%) and adherence rates (7.8-75\%). Participant satisfaction was assessed in three studies ${ }^{23,24,62}$ using the Client Satisfaction Questionnaire (CQS-8) and in two studies ${ }^{19,63}$ using other measures.

\section{Results of individual studies}

Primary outcomes: effectiveness of internet treatments

Effectiveness of internet-delivered treatments vs. a control condition
Are disorder-specific internet treatments effective, when compared to a control condition?

Out of seven RCTs, three studies ${ }^{19,63}$ compared guided 8-week disorder-specific iCBT to WLC.

Allen et al. ${ }^{19}$ investigated the efficacy of the 8-week Australian iCBT "Panic Program" in 63 adults with at least moderate PD symptom severity. This 5-lesson short version of the 6-lesson program ${ }^{65}$ involved brief telephone or email contacts with a therapist after the first two lessons. Results showed significant higher reductions in panic severity on the PDSSSR of iCBT compared to WLC, with large between-group effect sizes (Hedge's g=0.97). Self-report measures indicated more persons of the iCBT group (75\%) fell into the non-clinical range with a PDSS-SR score less than 7 compared to $\operatorname{WLC}(29 \%){ }^{19}$

Berger et al. ${ }^{23}$ evaluated the effects of guided 8-week iCBT transdiagnostic tailored and disorder-specific standardized iCBT (no names reported) compared to WLC in 132 Germanspeaking adults with at least one anxiety disorder $(33.3 \%$ with $\mathrm{PD} / \mathrm{PDA})$. The disorder-specific intervention was significantly more effective than WLC, with medium-to-large effect sizes ranging from Cohen's $\mathrm{d}=054$ (MIA) to $\mathrm{d}=0.97$ (ACQ), and significant clinical improvements of most participants with PD (62.5\%).

In a pragmatic RCT, van Ballegooijen et al. ${ }^{63}$ evaluated the 8-week Dutch iCBT "Don't Panic Online" in 126 adults with sub-clinical or mild panic symptoms (78.2\% with PD/PDA). In this 6-lesson iCBT program, trained Master-level students coached the iCBT group with weekly emails and support on demand. While IIT-analysis revealed no significant differences between the iCBT group and WLC on the PDSS-SR $(d=0.30)$, significant higher reductions in the iCBT-group were conversely identified in CC-analyses, with medium between-group effect size $(\mathrm{d}=0.73)$, and large within-effect size $(\mathrm{d}=1.23){ }^{63}$

Taken together, these disorder-specific iCBT appear being useful for motivated patients with clinically relevant PD symptoms.

Are transdiagnostic internet treatments effective, when compared to a control condition?

Out of five studies assessing transdiagnostic programs, two evaluated unguided and three studies guided internet treatments.

Berger et al. ${ }^{24}$ investigated the effects of a 9-week transdiagnostic tailored unguided iCBT ("velibra"). They randomized 139 German-speaking adults (64.0\% with PD/PDA, 45.3\% primary diagnosis) either to "CAU plus velibra" or "CAU only," whereas CAU involved a consultation with a physician in primary care. Program features contain daily automated messages, symptom tracking measures and personalization. As analyses of the PDA-subgroup showed, "velibra" was more effective than CAU only, with small between-group effect sizes ranging 
between $\mathrm{d}=0.22$ (MIB) and $\mathrm{d}=0.45$ (ACQ). Significantly more participants assigned to "velibra" (38.3\%) not met the diagnostic criteria for PD/PDA compared to participants receiving "CAU only" $(9.8 \%){ }^{24}$

Furthermore, Schröder et al. ${ }^{62}$ evaluated the effects of the unguided 4-week transdiagnostic German ConfID iCBT program ("CAU plus ConfID") compared to a "CAU" (WLC) in 179 adults (91.6\% with PD/PDA). Both participants assigned to ConfID or CAU/WLC were permitted to seek or maintain available therapies. Optional written feedback was provided by a psychologist and automated short message service (SMS). As expected, IIT-analyses demonstrated that this 4-module iCBT was more effective in reducing panic symptoms on the PAS than CAU, with medium effect size $\left(\eta_{p}{ }^{2}=0.071\right) .^{62}$

In the above outlined RCT by Berger et al..$^{23}$ the effects of guided 8-week iCBT transdiagnostic tailored $\mathrm{iCBT}$ were compared to WLC in 132 adults (29.5\% with PD/PDA in the tailored condition). Guidance was provided as email-contact with five Master students, a trained psychologist and a CBTtherapist. Significant improvements in panic symptoms of the transdiagnostic approache were identified, with small to large between-group effect sizes ranging from $\mathrm{d}=0.40$ (MIB) to $\mathrm{d}=0.88$ (ACQ), as well as more persons with clinically relevant improvements (69.2\%) compared to WLC (26.7\%). ${ }^{23}$

Oromendia et al. ${ }^{57}$ evaluated the effects of the 8-week transdiagnostic iCBT "Free from Anxiety" as Spanish adaptation. They randomized 77 adults with PD either to iCBT with scheduled psychological support (clinician-guided), iCBT with non-scheduled psychological support (self-guided) or WLC. As assumed, iCBT was significantly more effective in reducing panic symptoms on the PDSS-SR in both the guided ( $\mathrm{d}=$ 1.67) and unguided format $(\mathrm{d}=0.69)$, when compared to WLC.57

Finally, Ivanova et al. ${ }^{64}$ investigated the effects of an 8-week transdiagnostic Swedish internet-delivered ACT with a supplemented smartphone app ("ACT-smart") in 152 adults (25.7\% suffering primarily from PD). This 8-module internet-delivered ACT was, however, ineffective compared to WLC in reducing panic symptoms $(\mathrm{d}=0.05)$, but was effective in $\mathrm{SAD}$ and general anxiety. Nevertheless, the within-group effect sizes were large for the treated group $(\mathrm{d}=1.00)$ on the PDSSSR, and $25.9 \%$ of participants with PD clinically improved. ${ }^{64}$

Hence, the outlined five studies suggested that both guided and unguided transdiagnostic iCBT programs are effective.

To summarize, five out of seven RCTs (8 comparisons) confirmed the effectiveness of iCBT programs differing in conditions (e.g., support format, treatment approach, and duration). Moreover, all five studies assessing follow-up indicated the maintenance of treatment gains with large within-in effect sizes at 3-month, ${ }^{19} 6$ month ${ }^{23,24,57}$ and 12 months. ${ }^{64}$
Effectiveness of therapist-guided vs. unguided internet-delivered treatments

\section{Are guided treatments superior to unguided treatments?}

Three RCTs directly compared support formats.

In the aforementioned study on "Free from Anxiety" by Oromendia et al., ${ }^{57}$ participants of the clinician-guided -group were contacted weekly via telephone by a psychologist (total time spent per participnat, $\mathrm{M}=69.43 \mathrm{~min}, \mathrm{SD}=19.76$ ). Participants in the self-guided -group received weekly mails and support on demand. Analyses revealed significantly higher reductions of panic severity on the PDSS-SR in the guided group $(n=25)$ compared to the unguided group $(n=25)$, with a large between-group effect size ( $\mathrm{d}=1.18)$, and a significantly higher number of participants with clinically important improvements in panic symptoms ( $70.8 \%$ in the guided group vs. $20.8 \%$ in the unguided group). With-in effects were large (guided with $\mathrm{d}=1.30$; unguided with $\mathrm{d}=2.40$ ) and retained in both groups at the 6-month follow-up..$^{57}$

In contrast, two studies ${ }^{21,64}$ found no relevant impact of support format.

Fogliati et al. ${ }^{21}$ assessed 8-week clinician-guided and selfguided iCBT ("Panic Course" and "Wellbeing Course") in 145 adults from Australia (91\% with PD). Guidance was provided as weekly clinician-contact via telephone or secure email (total time spent per participant: $\mathrm{M}=36.79$ minutes, $\mathrm{SD}=21.35$ ). Participants in the self-guided iCBT were monitored and received weekly automated emails as well as support on demand. No significant differences between support formats on the PDSS-SR were identified; large within-in effects were found among persons with PD assigned to clinician-guided ( $\mathrm{n}=65, \mathrm{~d}=0.71)$ and self-guided iCBT $(\mathrm{n}=67$, $\mathrm{d}=1.09$ ). Improvements were maintained with large withinin effect sizes over the course of 24 months. ${ }^{21}$

In the above mentioned study on internet-delivered "ACTsmart" by Ivanova et al. ${ }^{64}$ guidance was provided twice per week by seven trained and weekly supervised Master students as written feedback via the app (time spent: about15 minutes per participant and week) in addition to automated messages. This study found no significant difference between participants with primary PD ( $\mathrm{n}=20$ at post-treatment) in the guided group $(n=9, d=1.18)$ and unguided group $(n=11$, $\mathrm{d}=0.99$ ) on the PDSS-SR. Effects were maintained at 12-month follow-up. ${ }^{64}$

Overall, the results indicate that both delivery modes are comparable in outcomes.

Effectiveness of transdiagnostic vs. disorder-specific internet-delivered treatments 


\section{Are transdiagnostic treatments superior to disorder-specific treatments?}

Two previously described RCTs ${ }^{21,23}$ investigated differences between both treatment approaches.

Berger et al. ${ }^{23}$ compared the effects of transdiagnostic tailored $(\mathrm{n}=44)$ with disorder-specific standardized iCBT $(\mathrm{n}=44)$. In the disorder-specific condition, $27.7 \%$ received access to PD/PDA-specific contents. Results showed no significant differences in PD-specific measures. Within-in effect sizes were large (transdiagnostic with $\mathrm{d}=1.09$ and disorder-specific with $\mathrm{d}=1.12$ ). However, the sample mainly consisted of patients with Social Anxiety Disorder (SAD; 86\%). ${ }^{23}$

Fogliati et al. ${ }^{21}$ assessed differences between the 5-session disorder-specific "Panic Course" ( $n=68$ with $P D)$ and the transdiagnostic "Wellbeing Course" ( $n=64$ with PD). They found no significant differences between the approaches on the PDSS-SR; both groups improved significantly from baseline to post-treatment (disorder-specific, $\mathrm{d}=0.79$; transdiagnostic, $\mathrm{d}=0.97) .^{21}$

Insofar, both studies did not indicate better outcomes of transdiagnostic approaches.

\section{Secondary outcomes: acceptability of internet treatments}

\section{Attrition and adherence}

As shown in Table 1, the attrition rates and adherence varied largely, with the lowest attrition rate was found in a guided ICBT (with 9.8\%). ${ }^{23}$ In contrast, three studies reported an attrition rate of more than 28\% (i.e., ConfID, Panic Program, Don't Panic Online). For instance, a high attrition rate (28.5\%) was found in the study on the 4-week unguided "ConfID,"62 in which only $52.8 \%$ indicated to interact more than once a week with "ConfID"-the most frequently stated reason for non-adherence was lack of time (47.4\%). In the RCT on the guided "Panic Program" (29.75\%), ${ }^{19}$ adherence amounted to $63 \%$. Finally, the highest attrition rate $(42.1 \%)$ was reported in the study on the guided 8-week "Don't Panic Online," ${ }^{33}$ in which only $8 \%$ of the participants finished all 6 lessons. Most frequently reported reasons for dropping out included time constraints, life events and symptom severity. ${ }^{63}$ Remarkly, in the unguided "velibra" program, ${ }^{24}$ the attrition rate was much lower (14\%), but the adherence rate amounting to $45.7 \%$ was poorer than in other studies with more dropouts.

Are guided internet treatments superior to unguided treatments in improving adherence?

The study on "Free from Anxiety" ${ }^{57}$ showed a moderate attrition rate (14.3\%), whereas, of the $20.8 \%$ dropping out of treatment, a significant lower rate was found in the clinician-guided group (8.3\%) compared to the self-guided group (33.3\%). Adherence was accordingly significantly higher in the clinicianguided-group, with $68 \%$ completing the entire program (vs. $44 \%$ of the self-guided group). ${ }^{57}$

Moreover, a positive effect of guidance was also found in another RCT: In the study on internet-delivered ACT by Ivanova et al. ${ }^{64}$ (with an attrition rate of $15.8 \%$ ), significantly more participants in the guided group completed all 8 modules (30\%) compared to the unguided group (7.8\%) in the app; conversely, the difference in adherence in the internet platform between guided group (40\%) and the unguided group (29.4\%) was not significant. Moreover, significant more entrances per week in the app were found in the guided group. ${ }^{64}$

In the RCT by Fogliati et al., ${ }^{21}$ the attrition rates ranged from $14 \%$ in the self-guided and $18 \%$ in the clinician-guided iCBT condition; adherence rates in terms of completion of all 5 lessons did not significantly differ between the self-guided (68\%) and the clinician-guided (69\%) condition.

Overall, two out of three studies showed benefits of guidance at least for adherence.

Are transdiagnostic internet treatments superior to disorder-specific treatments in improving adherence?

In the study on guided iCBT by Berger et al. ${ }^{23}$ (attrition rate of $9.8 \%$ ), adherence amounted to $75 \%$ in the transdiagnostic condition and $70.5 \%$ in the disorder-specific condition, with no significant difference in the average number of completed sessions ( 7 out of 8$){ }^{23}$

In the RCT by Fogliati et al., ${ }^{21}$ the attrition rates ranged from 11\% (disorder-specific "Panic Course") to 20\% (transdiagnostic "Wellbeing Course"); the adherence rate in terms of completion of all 5 lessons between 68\% (transdiagnostic) and $70 \%$ (disorder-specific) was also comparable across conditions.

\section{Participant satisfaction}

Treatment satisfaction was assessed in five RCTs, all with positive findings. Accordingly, three RCTs from Germanspeaking countries using the CSQ- $8^{23,24,62}$ indicated an overall relatively high participant satisfaction, in the unguided "velibra" (at the 6-month follow-up ${ }^{24}$ ) and in both guided tailored transdiagnostic and standardized disorder-specific iCBT, with no significant difference between both approaches. ${ }^{23}$ Furthermore, Schröder et al. ${ }^{62}$ used an adapted CSQ- 8 that indicated that most participants were satisfied with the quality of “ConfID” (73.6\%).

Different acceptability measures were used in both Australian studies. ${ }^{19,21}$ In the study by Allen et al., ${ }^{19} 93 \%$ of the participants indicated being mostly or very satisfied with the "Panic Program," and $87 \%$ reported being mostly or very confident 
to recommend the course to a friend with a similar problem.

Additionally, Fogliati et al. ${ }^{21}$ identified no significant differences in satisfaction ratings between support formats and approaches ("Wellbeing Course" and "Panic Course"); most (96-100\%) reported they would recommend the course to others and that it was worth their time (93-95\%).

Overall, there was a consistently high satisfaction across treatment modalities, whereas attrition and adherence rates differed.

\section{DISCUSSION}

\section{Summary of evidence and implications}

This review aimed at exploring the efficacy and acceptability of different internet-delivered treatment options for panic symptoms. Key findings and implications will be discussed next.

\section{Effectiveness of internet-delivered treatments}

Taken together, five out of seven RCTs showed significant improvements in panic symptoms in guided disorder-specific iCBT ${ }^{19,23}$ and in both unguided and guided transdiagnostic iCBT interventions, ${ }^{19,23,24,57,62,66}$ when compared to a control condition. These findings contribute to prior research targeting iCBT for anxiety disorders. ${ }^{14,30,38}$ Future studies should thus focus on determining specific therapeutic effects of iCBT, for instance, by using study designs combining active and passive control conditions. ${ }^{19,24}$ Another option could be to compare the efficacy of specific contents or modules such of internet treatments such as exposure or relaxation exercises in iCBT Moreover, the evidence base on predictors of outcome in iCBT for PD is limited. ${ }^{67}$ Interestingly, the study on "ConfID" ${ }^{\prime \prime 2}$ showed that a more positive attitude at baseline moderated therapeutic outcomes. A further investigation of the impact of pre-treatment attitudes in the response to iCBT could be therefore useful to decide, which patient might most likely benefit. ${ }^{68}$ Another interesting finding was that a good therapeutic working alliance could be established in different internet treatment approaches, ${ }^{23}$ which is consistent other research. ${ }^{6,69}$ Overall, findings suggest the helpfulness of internet treatments for PD patients with a preference for online selfhelp as stand-alone treatments and for those wishing to bridge waiting times for face-to-face therapy.

In contrast, IIT-analyses indicated no significant improvements in panic symptoms in two included RCTs. ${ }^{63,64}$ One of these studies assessed "Don't Panic Online" ${ }^{163}$ in a sample with mild or subclinical PD cases and proved to be effective in reducing panic symptoms only among program completers. In this context, van Ballegooijen et al..$^{63}$ noted the high proportion of comorbidity with Major Depressive Disorder (42.7\%) might have contributed to poor -adherence rates. Furthermore, Allen et al. ${ }^{19}$ argued that disorder-specific iCBT might be less helpful in subclinical PD. The other RCT ${ }^{64}$ with no meaningful effects for PD evaluated "ACT-smart" with a supplemented app. Ivanova et al. ${ }^{64}$ assumed the small proportion of PD cases in their study, the novelty of ACT protocols translated to internet-delivery and a probable superiority of iCBT over internet-delivered ACT for PD as possible reasons for non-significant findings. ${ }^{64}$ Indeed, the few published studies on internet-delivered ACT mostly targeted depression. ${ }^{17}$ Considering the scarce evidence base on the effectiveness of internet-delivered third-wave CBT programs on the one hand and the popularity of such trainings on the other hand, further research is recommended. ${ }^{15}$ Moreover, given the lack of evidence-based apps for $\mathrm{PD},{ }^{70}$ a further investigation of "ACT-smart" appears worthwhile. In addition, other third-wave CBT components such as mindfulness exercises have been successfully applied in several internet treatments like "velibra" or "Free from Anxiety."

Regarding support formats, only one $\mathrm{RCT}^{57}$ of three found significant improvements in panic symptoms and adherence rates in favor of guided treatments. This is in accordance with earlier research. ${ }^{22,71}$ Oromendia et al. ${ }^{57}$ assumed a positive impact of the scheduled support on participants' commitment to "Free from Anxiety," which might have increased adherence to treatment and therapeutic outcomes. Different reasons appear reasonable for the other two studies ${ }^{21,64}$ that found no significant difference between support formats in outcomes. Fogliati et al. ${ }^{21}$ argued that "new generation" iCBT work well as self-guided format, since they are grounded on well-established protocols, evaluated over several clinical trials and typically involve measures that aim at monitoring and engaging patients, such as automated messages. ${ }^{21} \mathrm{An}$ other possibility is the selection bias. For instance, in the study on the unguided "velibra," ${ }^{24}$ Berger et al. ${ }^{24}$ argued that the need for consulting a primary care physician might have increased the threshold for less motivated patients. This assumption of selection bias is also supported by the fact that in some studies ${ }^{21,57,62}$ only few participants in self-guided conditions accepted the offer of clinician-guidance on demand. Furthermore, although there were differences in the support quantity and modality in guided interventions, no patterns with outcomes or adherence appeared evident. Of the studies with a comparable average time spent per participant, some were effective, ${ }^{57}$ whereas others ${ }^{63}$ were not. In fact, the current state of research is incoherent, especially regarding the dose-dependency of support in treatment outcomes. ${ }^{22}$ A systematic review $^{22}$ found that guidance appears to be a helpful feature in internet treatments, but the impact they identified was smaller than stated earlier. Yet, studies on underlying process- 
es and specific components making different support formats effective and acceptable are scarce. ${ }^{21}$ For instance, in contrast to face-to-face treatments research indicates that the qualification of online-therapists appears to be irrelevant for outcomes. ${ }^{22,72}$ For decisions on suitable treatment options for PD, patients' preferences should be considered..$^{20}$ For instance, given a preference of many adults for self-help to manage milder psychological problems on one's own as a barrier to seek help face-to-face, ${ }^{73}$ unguided programs can be recommended for subclinical symptoms and prevention. ${ }^{22}$ It could be thus interesting to explore which subpopulations prefer guidance to provide stepped care models for PD. ${ }^{32}$ Surveys further suggest that many persons prefer therapist-guided IPT and faceto-face services in case of more severe emotional problems. ${ }^{74}$ Treatment preferences of patients with different symptomatology should be investigated to provide more or better suitable therapy options for specific subpopulations in routine care.

Furthermore, although of the most reviewed interventions were based on a transdiagnostic approach, only two studies $^{21,23}$ compared outcomes directly with the more established disorder-specific approach. Both studies showed no significant difference between the approaches. This is in contrast to findings of a review ${ }^{21}$ that indicated a superiority of disorderspecific over transdiagnostic iCBT for anxiety disorders. It would be therefore interesting to explore the role of perceived benefits across treatment approaches from the perspective of patients. ${ }^{35}$ Because tailoring allows for accurate assignment to treatment with respect to comorbid anxiety disorders, tailored transdiagnostic treatments have advantages for the dissemination of $\mathrm{iCBT}$ in routine care. ${ }^{24}$ Transdiagnostic treatments can work well as self-guided format, ${ }^{24,62}$ which is a clear benefit for the dissemination regarding treatment gaps. ${ }^{21}$ Thus, this combination appears efficient to provide additional treatment options.

\section{Acceptability of internet-delivered treatments}

Attrition rates varied largely across the RCTS. Interestingly, both the lowest $(9.8 \%)^{23}$ and the highest $(42.1 \%)^{63}$ dropout rate were found in different therapist-guided 8-week iCBT programs.

Relatively high attrition rates are commonly reported in iCBT trials for anxiety disorders. ${ }^{26}$ For instance, in reviews on internet-based treatments for different diagnoses dropout rates were between $2 \%$ and $89 \%$, with a weighted mean of $31 \%$ (19 studies), ${ }^{75}$ between $1 \%$ and $50 \%$ (23 studies), ${ }^{76}$ or between $0 \%$ and $55 \%$ (13 studies). ${ }^{29}$ In addition, adverse events as potential reasons for dropouts were not directly reported, which is a common issue in iCBT research. ${ }^{14,77}$

The present review also found variance in adherence rates, ranging from $7.8 \%$ to $75 \%$. For instance, in the study on the 5-lesson "Panic Program," 19 adherence (63\%) was lower than in a previous trial using a 6-lesson version (79\%). ${ }^{65}$ This is interesting, because the briefer version aimed at improving adherence; the same study reported that the 5-lesson "Panic Program" was also effective ( $\mathrm{g}=0.55$ ), when delivered in a primary care setting ( $\mathrm{n}=330$ patients), albeit the adherence rate was lower $(56.1 \%)$ than in the included $\mathrm{RCT} .{ }^{19}$ Clearing the relevance of iCBT length need further investigation. Consistent with other studies, ${ }^{60}$ two included studies ${ }^{62,63}$ identified lack of time as a barrier to complete iCBT programs. Ivanova et al. ${ }^{64}$ supposed that the relatively low degree of full adherence in the "ACT-smart" study could be attributed to multiple logins at different platforms and devices. Usability issues related to delivery modes (e.g., internet-based, computerized or smartphone-delivered) as reasons for non-adherence need detailed exploration. Moreover, minimal guidance may have improved adherence in two studies, ${ }^{64,57}$ and clinical effects in PD in one study. ${ }^{57}$ According to Mewton et al., ${ }^{12}$ guidance in iCBT is not needed for immediate improvements, but could be essential for the maintenance of clinical effects and adherence. In routine care, a combination of online and face-to-face format (i.e., blended treatments) can be useful as they can improve adherence or lower dropout rates. ${ }^{78}$

In accordance with prior research, ${ }^{38}$ five RCTs that assessed participant satisfaction endorsed a relatively high acceptance of internet treatment with different modalities. Earlier research ${ }^{14}$ indicated a high participant satisfaction in therapist-guided iCBT for anxiety disorders. However, in one included study, ${ }^{21}$ no difference in satisfaction was found between support formats. Furthermore, ratings stem from CCanalyses and might be thus biased towards higher satisfaction. Future studies should evaluate the (dis-) satisfaction of non-completers to improve the acceptability of Internet treatments. This could help identifying reasons for the discrepancy between high satisfaction and suboptimal adherence.

\section{Limitations}

This review has several limitations. On the study level, selfselection of participants limits the external validity. Samples mainly consisted of women who are at a higher risk for PD. ${ }^{2}$ Three studies with transdiagnostic scope had a low percentage of $\mathrm{PD} / \mathrm{PDA}$ cases in relation to SAD cases, although this reflects epidemiological distribution. ${ }^{23,24,64}$ Different confounding factors were not strictly controlled: although studies excluded severe mental illness, most made no restrictions made regarding ongoing pharmacotherapy (except for a stable dosage) or psychotherapy. Another issue is the limited availability of evidence-based programs. Furthermore, all studies used self-report measures. The absence of a control condition in the study by Fogliati et al. ${ }^{21}$ was another limitation. Attrition 
bias was another drawback in some studies. Nonetheless, the study quality was overall high. On the review basis, it should be noted that this was not a systematic review $\left(\right.$ c.f. $\left.{ }^{79}\right)$. Risk of bias is, for instance, increased due to the omission of a second reviewer. Additionally, the narrowed search strategy (e.g., time span) resulted in a selection of fewer studies. However, several studies on $\mathrm{iCBT}$ for PD published before December 2012 (e.g. ${ }^{65,80-84}$ ) were already analyzed in previous reviews. ${ }^{14}$ Another limitation is the restriction to few MeSH terms considering the inconsistent terminology in the literature. ${ }^{74}$ Thus, "internet treatment" was used as umbrella term for several delivery modes. Additionally, no MeSH terms for secondary outcomes were systematically searched. Also, only studies with mainly Caucasian samples were identified, albeit, to the author's knowledge, few iCBT publications have investigated crosscultural $^{85}$ or Asian samples. ${ }^{86}$ Moreover, seven out of 10 interventions were Non-English. Finally, consistent with earlier reviews, ${ }^{14,26,35}$ heterogeneity regarding the measurement hampered comparisons between studies.

\section{Conclusions}

Overall, this article illustrated different effective and wellaccepted evidence-based internet-delivered psychological treatments for panic disorder. Considering challenges related to treating comorbid anxiety disorders in times of treatment gaps, self-guided transdiagnostic iCBT programs appear to be efficient options for the large-scale dissemination in primary care. However, the discrepancy between high acceptability and slow uptake as well as the unclear role of patients' preferences require further investigation. Hence, clinicians should be informed about the latest advances in order to guide patients regarding the usefulness of internet-delivered treatments.

\section{Supplementary Materials}

The online-only Data Supplement is available with this article at https://doi.org/10.30773/pi.2018.06.26.

\section{REFERENCES}

1. Baxter AJ, Vos T, Scott KM, Ferrari AJ, Whiteford HA. The global burden of anxiety disorders in 2010. Psychol Med 2014;44:2363-2374.

2. Kessler RC, Chiu WT, Jin R, Ruscio AM, Shear K, Walters EE. The epidemiology of panic attacks, panic disorder, and agoraphobia in the National Comorbidity Survey Replication. Arch Gen Psychiatry 2006;63: 415-424.

3. Bandelow B, Michaelis S. Epidemiology of anxiety disorders in the 21st century. Dialogues Clin Neurosci 2015;17:327-335.

4. de Jonge P, Roest AM, Lim CC, Florescu SE, Bromet EJ, Stein DJ, et al. Cross-national epidemiology of panic disorder and panic attacks in the world mental health surveys. Depress Anxiety 2016;33:1155-1177.

5. Craske MG, Kircanski K, Epstein A, Wittchen HU, Pine DS, LewisFernández R, et al. Panic disorder: a review of DSM-IV panic disorder and proposals for DSM-V. Depress Anxiety 2010;27:93-112.

6. Bystritsky A, Khalsa SS, Cameron ME, Schiffman J. Current diagnosis and treatment of anxiety disorders. P T 2013;38:30-57.

7. Furukawa TA, Watanabe N, Churchill R. Psychotherapy plus antidepressant for panic disorder with or without agoraphobia: systematic review. Br J Psychiatry 2006;188:305-312.

8. Pompoli A, Furukawa TA, Imai H, Tajika A, Efthimiou O, Salanti G. Psychological therapies for panic disorder with or without agoraphobia in adults: a network meta-analysis. Cochrane Database Syst Rev 2016;4:CD011004.

9. Collins KA, Westra HA, Dozois DJ, Burns DD. Gaps in accessing treatment for anxiety and depression: challenges for the delivery of care. Clin Psychol Rev 2004;24:583-616.

10. Rickwood D, Bradford S. The role of self-help in the treatment of mild anxiety disorders in young people: an evidence-based review. Psychol Res Behav Manag 2012;5:25-36.

11. Musiat P, Tarrier N. Collateral outcomes in e-mental health: a systematic review of the evidence for added benefits of computerized cognitive behavior therapy interventions for mental health. Psychol Med 2014; 44:3137-3150.

12. Mewton L, Smith J, Rossouw P, Andrews G. Current perspectives on Internet-delivered cognitive behavioral therapy for adults with anxiety and related disorders. Psychol Res Behav Manag 2014;7:37-46.

13. Mayo-Wilson E, Montgomery P. Media-delivered cognitive behavioural therapy and behavioural therapy (self-help) for anxiety disorders in adults. Cochrane Database Syst Rev 2013:CD005330.

14. Olthuis JV, Watt MC, Bailey K, Hayden JA, Stewart SH. Therapist-supported Internet cognitive behavioural therapy for anxiety disorders in adults. Cochrane Database Syst Rev 2016;3:CD011565.

15. Apolinário-Hagen J, Salewski C. Internet-Based Cognitive Behavioral Therapy and Online Mindfulness Training for Panic Disorder. In: Kim YK, Editor. Panic Disorder: Assessment, Management and Research Insights. New York: NOVA SCIENCE, 2018, p.221-246.

16. Apolinário-Hagen J, Salewski C. Internet-Based Mindfulness-Based Cognitive Therapy for the Adjunctive Treatment of Major Depressive Disorder. In: Kim YK, Editor. Understanding Depression: Volume 2. Clinical Manifestations, Diagnosis and Treatment. Singapore: Springer Science and Business Media; Springer, 2018, p.299-309.

17. Brown M, Glendenning A, Hoon AE, John A. Effectiveness of web-delivered acceptance and commitment therapy in relation to mental health and well-being: a systematic review and meta-analysis. J Med Internet Res 2016;18:e221.

18. Johansson R, Frederick RJ, Andersson G. Using the internet to provide psychodynamic psychotherapy. Psychodyn Psychiatry 2013;41:513-540.

19. Allen AR, Newby JM, Mackenzie A, Smith J, Boulton M, Loughnan SA, et al. Internet cognitive-behavioural treatment for panic disorder: randomised controlled trial and evidence of effectiveness in primary care. BJPsych Open 2016;2:154-162.

20. Taylor CB. Panic disorder. BMJ 2006;332:951-955.

21. Fogliati VJ, Dear BF, Staples LG, Terides MD, Sheehan J, Johnston L, et al. Disorder-specific versus transdiagnostic and clinician-guided versus self-guided internet-delivered treatment for panic disorder and comorbid disorders: a randomized controlled trial. J Anxiety Disord 2016; 39:88-102.

22. Baumeister H, Reichler L, Munzinger M, Lin J. The impact of guidance on internet-based mental health interventions-a systematic review. Internet Interv 2014;1:205-215.

23. Berger T, Boettcher J, Caspar F. Internet-based guided self-help for several anxiety disorders: a randomized controlled trial comparing a tailored with a standardized disorder-specific approach. Psychotherapy (Chic) 2014;51:207-219.

24. Berger T, Urech A, Krieger T, Stolz T, Schulz A, Vincent A, et al. Effects of a transdiagnostic unguided Internet intervention ('velibra') for anxiety disorders in primary care: results of a randomized controlled trial. Psychol Med 2017;47:67-80.

25. Arnberg FK, Linton SJ, Hultcrantz M, Heintz E, Jonsson U. Internetdelivered psychological treatments for mood and anxiety disorders: a 
systematic review of their efficacy, safety, and cost-effectiveness. PLoS One 2014;9:e98118.

26. Peñate W, Fumero A. A meta-review of Internet computer-based psychological treatments for anxiety disorders. J Telemed Telecare 2016;22: 3-11.

27. Barak A, Hen L, Boniel-Nissim M, Shapira N. A comprehensive review and a meta-analysis of the effectiveness of internet-based psychotherapeutic interventions. J Technol Hum Serv 2008;26:109-160.

28. Cuijpers P, Marks IM, van Straten A, Cavanagh K, Gega L, Andersson G. Computer-aided psychotherapy for anxiety disorders: a meta-analytic review. Cogn Behav Ther 2009;38:66-82.

29. Andersson G, Cuijpers P, Carlbring P, Riper H, Hedman E. Guided Internet-based vs. face-to-face cognitive behavior therapy for psychiatric and somatic disorders: a systematic review and meta-analysis. World Psychiatry 2014;13:288-295.

30. Carlbring P, Andersson G, Cuijpers P, Riper H, Hedman-Lagerlöf E. Internet-based vs. face-to-face cognitive behavior therapy for psychiatric and somatic disorders: an updated systematic review and meta-analysis. Cogn Behav Ther 2018;47:1-18.

31. Hedman E, Ljótsson B, Rück C, Bergström J, Andersson G, Kaldo V, et al. Effectiveness of internet-based cognitive behaviour therapy for panic disorder in routine psychiatric care. Acta Psychiatr Scand 2013;128: 457-467.

32. Nordgreen T, Haug T, Öst LG, Andersson G, Carlbring P, Kvale G, et al. Stepped care versus direct face-to-face cognitive behavior therapy for social anxiety disorder and panic disorder: a randomized effectiveness trial. Behav Ther 2016;47:166-183.

33. Saddichha S, Al-Desouki M, Lamia A, Linden IA, Krausz M. Online interventions for depression and anxiety - a systematic review. Health Psychol Behav Med 2014;2:841-881.

34. Andrews G, Cuijpers P, Craske MG, McEvoy P, Titov N. Computer therapy for the anxiety and depressive disorders is effective, acceptable and practical health care: a meta-analysis. PLoS One 2010;5:e13196.

35. Newby JM, Twomey C, Yuan Li SS, Andrews G. Transdiagnostic computerised cognitive behavioural therapy for depression and anxiety: a systematic review and meta-analysis. J Affect Disord 2016;199:30-41.

36. Păsărelu CR, Andersson G, Bergman Nordgren L, Dobrean A. Internetdelivered transdiagnostic and tailored cognitive behavioral therapy for anxiety and depression: a systematic review and meta-analysis of randomized controlled trials. Cogn Behav Ther 2017;46:1-28.

37. Andersen P, Toner P, Bland M, McMillan D. Effectiveness of transdiagnostic cognitive behaviour therapy for anxiety and depression in adults: a systematic review and meta-analysis. Behav Cogn Psychother 2016; 44:673-690.

38. Andrews G, Basu A, Cuijpers P, Craske MG, McEvoy P, English CL, et al. Computer therapy for the anxiety and depression disorders is effective, acceptable and practical health care: an updated meta-analysis. J Anxiety Disord 2018;55:70-78.

39. Methley AM, Campbell S, Chew-Graham C, McNally R, Cheraghi-Sohi S. PICO, PICOS and SPIDER: a comparison study of specificity and sensitivity in three search tools for qualitative systematic reviews. BMC Health Serv Res 2014;14:579.

40. Dettori JR. Loss to follow-up. Evid Based Spine Care J 2011;2:7-10.

41. Ciuca AM, Berger T, Crişan LG, Miclea M. Internet-based treatment for Romanian adults with panic disorder: protocol of a randomized controlled trial comparing a Skype-guided with an unguided self-help intervention (the PAXPD study). BMC Psychiatry 2016;16:6.

42. González-Robles A, García-Palacios A, Baños R, Riera A, Llorca G, Traver F, et al. Effectiveness of a transdiagnostic internet-based protocol for the treatment of emotional disorders versus treatment as usual in specialized care: study protocol for a randomized controlled trial. Trials 2015;16:488.

43. Ebenfeld L, Kleine Stegemann S, Lehr D, Ebert DD, Jazaieri H, van Ballegooijen W, et al. Efficacy of a hybrid online training for panic symptoms and agoraphobia: study protocol for a randomized controlled tri- al. Trials 2014;15:427.

44. Lindner P, Ivanova E, Ly KH, Andersson G, Carlbring P. Guided and unguided CBT for social anxiety disorder and/or panic disorder via the Internet and a smartphone application: study protocol for a randomised controlled trial. Trials 2013;14:437.

45. Bruinsma A, Kampman M, Exterkate CC, Hendriks GJ. Een verkennende studie naar blended cognitieve gedragstherapie voor paniekstoornis: resultaten en patiëntervaringen. Tijdschr Psychiatr 2016;58: 361-370.

46. Vigerland S, Ljótsson B, Thulin U, Öst LG, Andersson G, Serlachius E. Internet-delivered cognitive behavioural therapy for children with anxiety disorders: a randomised controlled trial. Behav Res Ther 2016;76: 47-56.

47. Manita C, Barroso R, Eher R, Boer D. Criminal policies in sexual violence: from research to legislation and treatment. 13th conference of the International Association for the Treatment of Sexual Offenders (IATSO), September 3-6, 2014, Porto, Portugal. Forensische Psychiatrie und Psychotherapie 2014;21:17-181.

48. Rozental A, Boettcher J, Andersson G, Schmidt B, Carlbring P. Negative effects of internet interventions: a qualitative content analysis of patients' experiences with treatments delivered online. Cogn Behav Ther 2015;44:223-236.

49. Andersson G, Hedman E. Effectiveness of guided internet-based cognitive behavior therapy in regular clinical settings. Verhaltenstherapie 2013; 23:140-148.

50. Riccardi CJ, Korte KJ, Schmidt NB. False safety behavior elimination therapy: a randomized study of a brief individual transdiagnostic treatment for anxiety disorders. J Anxiety Disord 2017;46:35-45.

51. Dear BF, Staples LG, Terides MD, Fogliati VJ, Sheehan J, Johnston L, et al. Transdiagnostic versus disorder-specific and clinician-guided versus self-guided internet-delivered treatment for Social Anxiety Disorder and comorbid disorders: a randomized controlled trial. J Anxiety Disord 2016;42:30-44.

52. Dear BF, Staples LG, Terides MD, Karin E, Zou J, Johnston L, et al. Transdiagnostic versus disorder-specific and clinician-guided versus self-guided internet-delivered treatment for generalized anxiety disorder and comorbid disorders: a randomized controlled trial. J Anxiety Disord 2015;36:63-77.

53. Titov N, Dear BF, Staples LG, Terides MD, Karin E, Sheehan J, et al. Disorder-specific versus transdiagnostic and clinician-guided versus self-guided treatment for major depressive disorder and comorbid anxiety disorders: a randomized controlled trial. J Anxiety Disord 2015;35: 88-102.

54. El Alaoui S, Hedman E, Ljótsson B, Bergström J, Andersson E, Rück C, et al. Predictors and moderators of internet- and group-based cognitive behaviour therapy for panic disorder. PLoS One 2013;8:e79024.

55. Haug T, Nordgreen T, Öst LG, Kvale G, Tangen T, Andersson G, et al. Stepped care versus face-to-face cognitive behavior therapy for panic disorder and social anxiety disorder: predictors and moderators of outcome. Behav Res Ther 2015;71:76-89.

56. Johnston L, Titov N, Andrews G, Dear BF, Spence J. Comorbidity and internet-delivered transdiagnostic cognitive behavioural therapy for anxiety disorders. Cogn Behav Ther 2013;42:180-192.

57. Oromendia P, Orrego J, Bonillo A, Molinuevo B. Internet-based selfhelp treatment for panic disorder: a randomized controlled trial comparing mandatory versus optional complementary psychological support. Cogn Behav Ther 2016;45:270-286.

58. Christoforou M, Sáez Fonseca JA, Tsakanikos E. Two novel cognitive behavioral therapy-based mobile apps for agoraphobia: randomized controlled trial. J Med Internet Res 2017;19:e398.

59. Mathiasen K, Riper H, Ehlers LH, Valentin JB, Rosenberg NK. Internetbased CBT for social phobia and panic disorder in a specialised anxiety clinic in routine care: results of a pilot randomised controlled trial. Internet Interv 2016;4:92-98.

60. Kok RN, van Straten A, Beekman AT, Cuijpers P. Short-term effective- 
ness of web-based guided self-help for phobic outpatients: randomized controlled trial. J Med Internet Res 2014;16:e226.

61. Boettcher J, Aström V, Påhlsson D, Schenström O, Andersson G, Carlbring P. Internet-based mindfulness treatment for anxiety disorders: a randomized controlled trial. Behav Ther 2014;45:241-253.

62. Schröder J, Jelinek L, Moritz S. A randomized controlled trial of a transdiagnostic Internet intervention for individuals with panic and phobias - one size fits all. J Behav Ther Exp Psychiatry 2017;54:17-24.

63. van Ballegooijen W, Riper H, Klein B, Ebert DD, Kramer J, Meulenbeek P, et al. An Internet-based guided self-help intervention for panic symptoms: randomized controlled trial. J Med Internet Res 2013;15: e154.

64. Ivanova E, Lindner P, Ly KH, Dahlin M, Vernmark K, Andersson G, et al. Guided and unguided Acceptance and Commitment Therapy for social anxiety disorder and/or panic disorder provided via the Internet and a smartphone application: a randomized controlled trial. J Anxiety Disord 2016;44:27-35.

65. Wims E, Titov N, Andrews G, Choi I. Clinician-assisted Internet-based treatment is effective for panic: a randomized controlled trial. Aust $\mathrm{N} \mathrm{Z}$ J Psychiatry 2010;44:599-607.

66. Berger $\mathrm{T}$. The therapeutic alliance in internet interventions: a narrative review and suggestions for future research. Psychother Res 2017;27: 511-524.

67. Nordgreen T, Standal B, Mannes H, Haug T, Sivertsen B, Carlbring P, et al. Guided self-help via internet for panic disorder: dissemination across countries. Comput Hum Behav 2010;26:592-596.

68. Schröder J, Sautier L, Kriston L, Berger T, Meyer B, Späth C, et al. Development of a questionnaire measuring Attitudes towards Psychological Online Interventions-the APOI. J Affect Disord 2015;187:136-141.

69. Pihlaja S, Stenberg J, Joutsenniemi K, Mehik H, Ritola V, Joffe G. Therapeutic alliance in guided internet therapy programs for depression and anxiety disorders - a systematic review. Internet Interv 2018;11:1-10.

70. van Singer M, Chatton A, Khazaal Y. Quality of smartphone apps related to panic disorder. Front Psychiatry 2015;6:96.

71. Andersson G, Cuijpers P. Internet-based and other computerized psychological treatments for adult depression: a meta-analysis. Cogn Behav Ther 2009;38:196-205.

72. Shim M, Mahaffey B, Bleidistel M, Gonzalez A. A scoping review of human-support factors in the context of Internet-based psychological interventions (IPIs) for depression and anxiety disorders. Clin Psychol Rev 2017;57:129-140.

73. Andrade LH, Alonso J, Mneimneh Z, Wells JE, Al-Hamzawi A, Borges
G, et al. Barriers to mental health treatment: results from the WHO World Mental Health surveys. Psychol Med 2014;44:1303-1317.

74. Apolinário-Hagen J, Kemper J, Stürmer C. Public acceptability of emental health treatment services for psychological problems: a scoping review. JMIR Mental Health 2017;4:e10.

75. Melville KM, Casey LM, Kavanagh DJ. Dropout from Internet-based treatment for psychological disorders. Br J Clin Psychol 2010;49:455471.

76. Christensen H, Griffiths KM, Farrer L. Adherence in internet interventions for anxiety and depression. J Med Internet Res 2009;11:e13.

77. Rozental A, Andersson G, Boettcher J, Ebert D, Cujipers P, Knaevelsrud $\mathrm{C}$, et al. Consensus statement on defining and measuring negative effects of Internet interventions. Internet Interv 2014;1:12-19.

78. Erbe D, Eichert HC, Riper H, Ebert DD. Blending face-to-face and internet-based interventions for the treatment of mental disorders in adults: systematic review. J Med Internet Res 2017;19:e306.

79. Moher D, Liberati A, Tetzlaff J, Altman DG; PRISMA Group. Preferred reporting items for systematic reviews and meta-analyses: the PRISMA statement. PLoS Med 2009;6:e1000097.

80. Carlbring P, Ekselius L, Andersson G. Treatment of panic disorder via the internet: a randomized trial of CBT vs. applied relaxation. J Behav Ther Exp Psychiatry 2003;34:129-140.

81. Carlbring P, Westling BE, Ljungstrand P, Ekselius L, Andersson G. Treatment of panic disorder via the internet: a randomized trial of a self-help program. Behav Ther 2001;32:751-764.

82. Kiropoulos LA, Klein B, Austin DW, Gilson K, Pier C, Mitchell J, et al. Is internet-based CBT for panic disorder and agoraphobia as effective as face-to-face CBT? J Anxiety Disord 2008;22:1273-1284.

83. Klein B, Richards JC, Austin DW. Efficacy of internet therapy for panic disorder. J Behav Ther Exp Psychiatry 2006;37:213-238.

84. Silfvernagel K, Carlbring P, Kabo J, Edström S, Eriksson J, Månson L, et al. Individually tailored internet-based treatment for young adults and adults with panic attacks: randomized controlled trial. J Med Internet Res 2012;14:e65.

85. Kayrouz R, Dear BF, Karin E, Fogliati VJ, Titov N. A pilot study of a clinician-guided internet-delivered cognitive behavioural therapy for anxiety and depression among Arabs in Australia, presented in both English and Arabic languages. Internet Interv 2016;5:5-11.

86. Seol SH, Kwon JS, Kim YY, Kim SN, Shin MS. Internet-based cognitive behavioral therapy for obsessive-compulsive disorder in Korea. Psychiatry Investig 2016;13:373-382. 